\title{
Large Thermoelectric Effects and Inelastic Scattering in Unconventional Superconductors
}

\author{
Mikael Fogelström* and Tomas Löfwander ${ }^{\dagger}$ \\ *Applied Quantum Physics, MC2, Chalmers University of Technology, S-412 96 Göteborg, Sweden \\ †Institut für Theoretische Festkörperphysik, Universität Karlsruhe, 76128 Karlsruhe, Germany
}

\begin{abstract}
The thermoelectric coefficient $\eta(T)$ in unconventional superconductors is enhanced below $T_{c}$ by intermediate strength impurity scattering that is intrinsically particle-hole asymmetric. We compute $\eta(T)$ for a strong-coupling d-wave superconductor and investigate the effects of inelastic scattering originating from electron-boson interactions. We show that $\eta(T)$ is severely suppressed at temperatures just below $T_{c}$ by a particle-hole symmetric inelastic scattering rate. At lower temperatures inelastic scattering is frozen out and $\eta(T)$ recovers and regains its large amplitude. In the limit $T \rightarrow 0$, we have $\eta(T) \sim \eta_{0} T+\mathscr{O}\left[T^{3}\right]$, where the slope $\eta_{0}$ contains information about the Drude plasma frequency, the details of impurity scattering, and the change in effective mass by electron-boson interactions. In this limit $\eta(T)$ can be used as a probe, complementary to the universal heat and charge conductivities, in investigations of the nature of nodal quasiparticles.
\end{abstract}

Low-temperature transport measurements have provided a wealth of information about nodal quasiparticles in unconventional superconductors [1, 2]. Thermal conductivity is of particular importance, because theory predicts universality in the sense that the low-temperature asymptotic does not depend on the properties of the impurity potential [3]. This prediction has been confirmed experimentally [1]. However, there are some difficulties in analysing the low-temperature thermal conductivity. First, the leading $T^{2}$-dependence of the electronic contribution to $\kappa(T) / T$ is masked by a phonon contribution with the same $T^{2}$-dependence [2]. Second, experiments are often done on ultra-clean samples, which means that the $T^{2}$ power law of $\kappa(T) / T$ holds only in a small temperature bracket [4].

In a recent paper we discussed how isotropic elastic scattering by impurities of intermediate strength, i.e described by a phase shift $0<\delta_{0}<\frac{\pi}{2}$, gives rise to an electron-hole asymmetric scattering time and consequently a large non-universal thermoelectric response [5]. A careful study of the thermoelectric coefficient in the low-temperature regime would reveal information about the bare elastic scattering rate and potential strength. This information is hard to extract from thermal conductivity data. In this report we examine the interplay between elastic and inelastic scattering and show how it affects the thermoelectric coefficient.

The thermoelectric coefficient, $\eta(T)$, is defined as

$$
\delta \vec{j}_{e}=-\eta(T) \nabla T=2 \mathscr{N}_{f} \int d \vec{p}_{f} \int \frac{d \varepsilon}{4 \pi i} e \vec{v}_{f} \delta g^{K} .
$$

The quasiclassical propagator $\delta \hat{g}^{K}$ has a closed form in which the self-consistently computed equilibrium Green function $\hat{g}_{0}^{R}$ and the self-energy $\hat{\Sigma}^{R}$ serve as input (see Graf et al. [3] and Ref. [5] for details). In the present study we consider a composite self-energy in particlehole space $\hat{\Sigma}^{R}=\hat{\Sigma}_{\text {imp }}^{R}+\hat{\Sigma}_{\text {in }}^{R}$, where the impurity selfenergy $\hat{\Sigma}_{\text {imp }}^{R}$ is diagonal with $\Sigma_{3 \text {,imp }}^{R}$ and $\Sigma_{0, \text { imp }}^{R}$ being its particle-hole symmetric and anti-symmetric parts, respectively. The self-energy $\hat{\Sigma}_{\text {in }}^{R}$ includes the effects of inelastic electron-boson scattering, but we also assume that this interaction mediates the pairing. Below, $\hat{W}^{R}$ is the off-diagonal component, i.e the usual strong-coupling function related to the energy dependent gap as $\hat{\Delta}^{R}(\varepsilon)=$ $\hat{W}^{R}(\varepsilon) / Z(\varepsilon)$. Contrary to the impurity self-energy the diagonal part of the inelastic self-energy is particle-hole symmetric as $\Sigma_{0, \text { in }}^{R}=0$. Finally, $Z(\varepsilon)$ is the energyrenormalization function defined by the scattering renormalized energy $\tilde{\varepsilon}^{R}=Z^{R}(\varepsilon) \varepsilon=\varepsilon-\Sigma_{3, \text { imp }}^{R}-\Sigma_{3, \text { in }}^{R}$. This model is the same as we used in our study of the thermal conductivity [4]. With this input we use Ref. [5] and write down the response function

$$
\begin{aligned}
\eta_{i j}(T)=- & \frac{e}{4 T^{2}} \int d \varepsilon \varepsilon \operatorname{sech}^{2} \frac{\varepsilon}{2 T} \int d \vec{p}_{f}\left[v_{f, i} v_{f, j}\right] \\
& \times \frac{\mathscr{N}\left(\vec{p}_{f}, \varepsilon\right) \mathfrak{I} \Sigma_{0, i m p}^{R}(\varepsilon)}{\left[\Re \Omega^{R}\left(\vec{p}_{f} ; \varepsilon\right)\right]^{2}-\left[\mathfrak{I} \Sigma_{0, i m p}^{R}(\varepsilon)\right]^{2}},
\end{aligned}
$$

where $\mathscr{N}\left(\vec{p}_{f}, \varepsilon\right)=-\mathscr{N}_{f} \mathfrak{I}\left[\tilde{\varepsilon}^{R} / \Omega^{R}\left(\vec{p}_{f} ; \varepsilon\right)\right]$ is the density of states, and $\Omega^{R}=\sqrt{\left|W^{R}\left(\vec{p}_{f}, \varepsilon\right)\right|^{2}-\left(\tilde{\varepsilon}^{R}\right)^{2}}$. Note that $\eta(T)$ is directly proportional to the imaginary part of the 

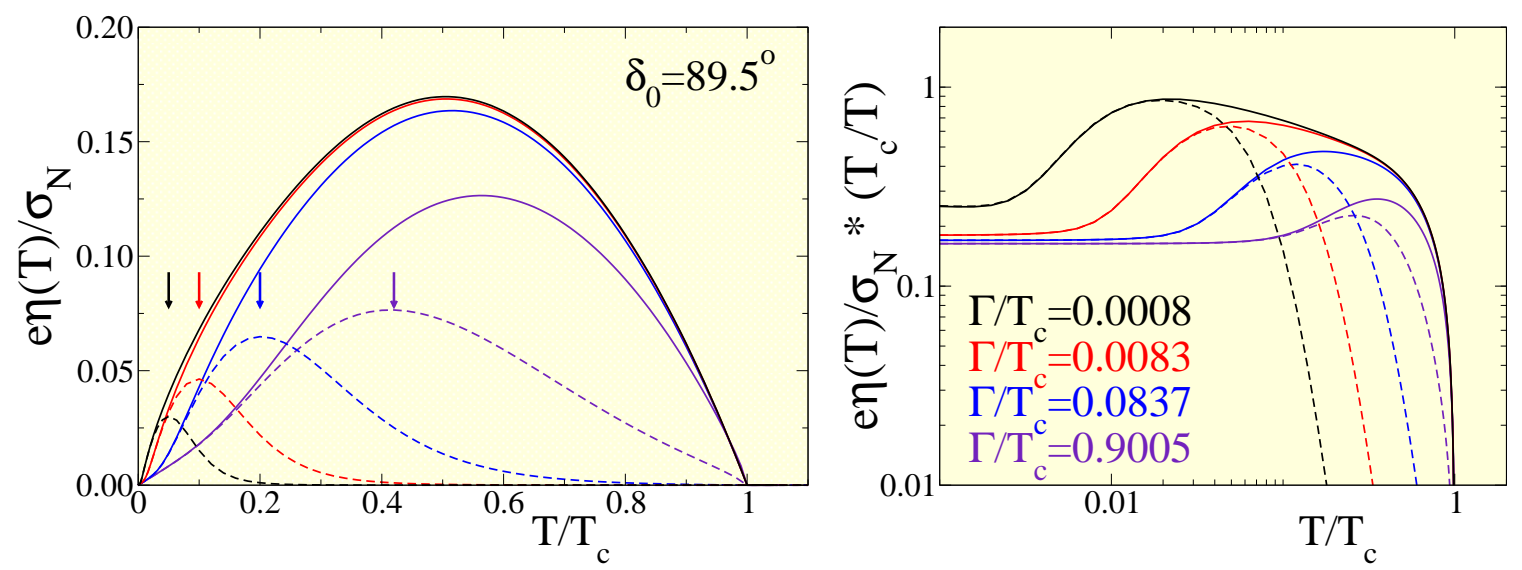

FIGURE 1. Thermoelectric coefficient, $\eta(T)$, calculated for four orders of magnitude in scattering rate, as indicated in the right panel. The dashed lines are results of a self-consistent calculation with the inelastic scattering model introduced in Ref. [4]. The full lines are the corresponding results obtained with an effective model where the inelastic part is included only as an effective mass via $m^{*} / m=1+\lambda_{\text {in }}[4]$. The temperature dependence obtained with the effective model is the same as within weak-coupling theory. In the left panel $\eta(T)$ is scaled by the normal state Drude conductance, $\sigma_{N}=e^{2} v_{f}^{2} \mathscr{N}_{f} / 2 \Gamma$, where $v_{f}, \mathscr{N}_{f}$, and $\Gamma$ are effective values of respective bare constants. The arrows indicate the cross-over, $T^{*}$, between the two scattering modes. In the right panel we show the temperature dependence of $\eta(T) / T$ with emphasis on the low-temperature regime where the leading $T$-dependence is $\eta(T) / T \approx \eta_{0}$. The constant, $\eta_{0}$, is non-universal since it explicitly depends on the ratio of $\partial \mathfrak{I} \Sigma_{0, \text { imp }}^{R}(\varepsilon) /\left.\partial \varepsilon\right|_{\varepsilon=0}$ and $\mathfrak{I} \Sigma_{3, \text { imp }}^{R}(\varepsilon=0)$.

particle-hole asymmetric part $\mathfrak{I} \Sigma_{0, \text { imp }}^{R}$, which is an odd function of energy.

In Figure 1 we show $\eta(T)$ for a strong-coupling dwave superconductor. The results are compared with an effective model-calculation where only the mass renormalization by inelastic scattering is accounted for, as described in Ref. [4]. As seen in Figure 1] inelastic scattering affects $\eta(T)$ much in the same way as it does the thermal conductivity reported in Ref. [4], i.e it dominates at temperatures $T \leq T_{c}$ but freezes out at low $T \ll T_{c}$ where instead elastic scattering limits the transport. We separate the two regimes where the two types of scattering dominate by introducing a cross-over temperature $T^{*}$, defined as the temperature where $\eta(T)$ has its maximium. The value of $T^{*}$ depends on the elastic scattering rate $\Gamma$ for a given electron-boson coupling spectra. The suppression of $\eta(T)$ in the interval $T^{*} \lesssim T \leq T_{c}$ comes from that in our model inelastic scattering does not break particle-symmetry and hence reduces the weight of the kernel in the integral in Eq. (2).

In the low- $T$ regime $T \ll\left|\mathfrak{I} \Sigma_{3, \text { imp }}^{R}(0)\right|$, we obtain

$$
\frac{\eta(T)}{T}=e \frac{\pi^{2}}{3} \frac{2 \mathscr{N}_{f} v_{f}^{2}}{\pi \mu \Delta_{0}}\left|\frac{\partial_{\varepsilon} \mathfrak{I} \Sigma_{0, \text { imp }}^{R}(\varepsilon)}{\mathfrak{I} \Sigma_{3, \text { imp }}^{R}(\varepsilon)}\right|_{\mathcal{E}=0}+\mathscr{O}\left[T^{2}\right],
$$

where $\Delta_{0}$ is the spectroscopic gap, and $\mu=$ $\left(1 / \Delta_{0}\right)|d \Delta(\phi) / d \phi|_{\phi_{\text {node }}}$ is the opening rate of the gap function at the node. In analogy with the thermal conductivity [4], the remaining effect at $T \ll T^{*}$ of inelastic scattering is a modification of the $T \rightarrow 0$ asymptotic. When we write explicitly $\mathscr{N}_{f} \rightarrow \mathscr{N}_{f}^{*}=\mathscr{N}_{f}^{0}\left(1+\lambda_{\text {in }}\right)$ and $v_{f} \rightarrow v_{f}^{*}=v_{f}^{0} /\left(1+\lambda_{\text {in }}\right)$ in Eq. (3), one factor $1+\lambda_{\text {in }}$ remains in the denominator. Within the bare theory, this result can be traced back to that the spectroscopic gap $\Delta_{0}$ in the weak-coupling limit is replaced by the strong coupling off-diagonal function $W(0)$.

In summary, we have discussed the temperaturedependence of the thermoelectric effect that results from the interplay of inelastic electron-boson scattering and elastic impurity scattering. At high temperatures, $T>$ $T^{*}$, particle-hole symmetric inelastic scattering dominates and the thermoelectric coefficient is suppressed. On the other hand, at low temperatures, $T<T^{*}$, particlehole asymmetric elastic impurity scattering dominates and the thermoelectric coefficient is enhanced.

We gratefully acknowledge financial support from the Swedish Research Council (M.F), and the EC under the Spintronics Network RTN2-2001-00440 (T.L).

\section{REFERENCES}

1. L. Taillefer et al, Phys. Rev. Lett. 79, 483 (1997); S. Nakamae et al. , Phys. Rev. B 63, 184509 (2001); J. Takeya, Y. Ando, S. Komiya, and X. F. Sun, Phys. Rev. Lett. 88, 077001 (2002).

2. R. W. Hill, et al, Phys. Rev. Lett. 92, 027001 (2004).

3. M. J. Graf, S.-K. Yip, J. A. Sauls, and D. Rainer, Phys. Rev. B 53, 15147 (1996); A. Durst and P. A. Lee, Phys. Rev. B 62, 1270 (2000).

4. T. Löfwander and M. Fogelström, cond-mat/0503391 accepted for publication in Phys. Rev. Lett.

5. T. Löfwander and M. Fogelström, Phys. Rev. B 70, 024515 (2004). 